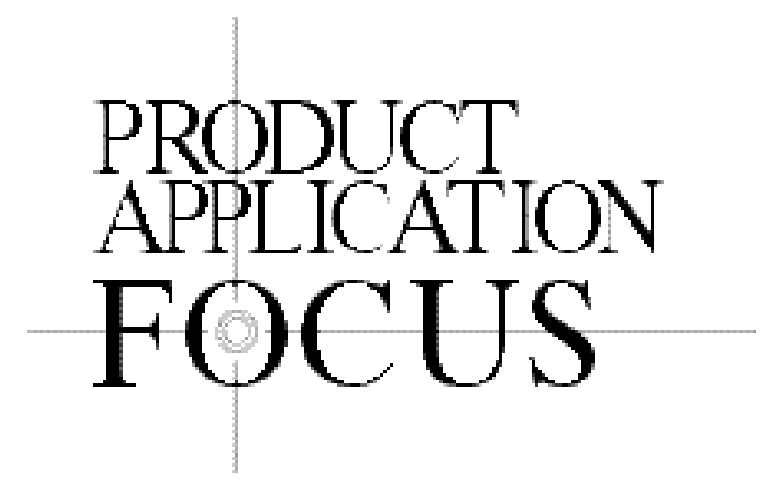

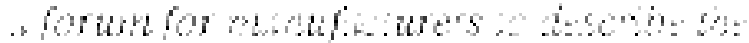

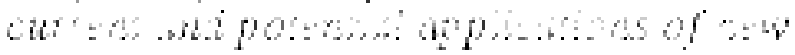

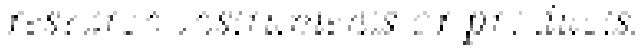

\title{
Bicistronic Expression of Ecdysone-Inducible Receptors in Mammalian Cells
}

\author{
Denise L. Wyborski, John C. Bauer, and Peter Vaillancourt \\ Stratagene Cloning Systems, La Jolla, CA, USA
}

BioTechniques 31:618-624 (September 2001)

\begin{abstract}
The recent emergence of inducible expression systems for mam malian cells has greatly facilitated the in vivo analysis of gene function. The ecdysone-inducible expression system is particularly attractive because of (i) extremely low basal expression and high-level induced expression, (ii) the lack of pleiotropic effects caused by the inducer or activator, and (iii) the rapid penetrance and clearance of the inducer. Here, we describe an improved receptor expression vector. The required ecdysone receptor proteins $(V g E c R$ and $R X R)$ are co-expressed from a bicistronic cytomegalovirus (CMV) expression cassette in the vector $p E R V 3$. The CMV promoter in this vector can be readily replaced with a cell type-specific promoter of interest. Using the ecdysone analogs, muristerone A or ponasterone A, induction ratios of up to three orders of magnitude were attained in the transient transfection assays and in a cell line stably transformed with both $p E R V 3$ and an ecdysone-inducible reporter vector. Fine control of luciferase expression was achieved by varying both the induction time and inducer concentration. Here, we describe a set of cell lines stably transformed with the vector pERV3, in which the ecdysone receptors are expressed at optimal levels for the high-level induction of gene expression.
\end{abstract}

\section{INTRODUCTION}

DNA vector-based systems that allow fine control of gene expression in mammalian cells have become invaluable in the study of biological processes for which the time or dose of gene expression is critical to gene function. These systems have been successfully used for the controlled expression of toxic genes, to induce gene targeting, and to express antisense RNA $(9,11)$. Inducible systems are currently being used by pharmaceutical companies to facilitate the screening for in- hibitors of clinically relevant biological pathways, and applications for controlled expression of therapeutic genes in vivo are being explored.

The ecdysone-inducible expression system $(3,8,9)$ is particularly attractive because of $(i)$ negligible background expression from the naturally repressed ecdysone-inducible promoter in the absence of the inducer, (ii) rapid, high-level induction upon administration of the inducer, (iii) lack of cross-talk between the inducible system and endogenous cellular pathways, (iv) rapid penetrance and clearance of the inducer in all tissues, allowing fine temporal control of gene expression, and $(v)$ precise control of expression levels in response to varying inducer concentrations. The system com prises an expression vector in which the gene of interest is inserted downstream of an ecdysone-inducible promoter, and one or two additional vectors from which the requisite ecdysone receptor proteins RXR and $\mathrm{VgEcR}$ are produced from separate expression cassettes $(8,10)$. Most applications require the separate production of stable cell lines or transgenic animals with the receptor expression vectors, screening for clones in which the receptors are expressed at optimal levels, and then the introduction of the inducible vector in a second round of stable cell line production or by mating with a mouse harboring the inducible transgene.

For many applications, it is desirable to express the receptors from a cell type-specific promoter to regulate the expression of a gene of interest in an appropriate tissue. This feature is of particular importance for the production of transgenic animals, in which every cell type will contain the receptor and inducible expression cassettes. Here, we describe the ecdysone receptor expression vector pERV3, from which both VgEcR and RXR are co-expressed from a single dicistronic mRNA transcribed from the cytomegalovirus (CMV) promoter (Figure 1A). The major advantage to co-ex- 
pressing the receptor proteins from a single promoter is that the promoter can be readily replaced with a cell type-specific promoter of interest in a single subcloning event, as opposed to replacing each of two promoters in separate receptor protein expression cassettes. Another potential advantage is that the use of a single transcription unit may increase the likelihood of uniform co-expression of the proteins in a variety of cell types $(1,4)$, compared with two-plasmid systems $(8)$, for which position effects of chromosomal integration may interfere with the efficient co-expression of both proteins, or with vectors containing independent receptor expression cassettes employing two different promoters that may be differentially regulated in different cell types (10). However, the importance of this latter advantage is not completely clear, in light of reports of cell type to cell type inconsistencies in the translation from the encephalomyocarditis virus (EMCV) internal ribosome entry site (IRES) (1), which is employed in the pERV3 vector for expression of the RXR protein from the downstream open reading frame in the bicistronic transcript.

In this report, we demonstrate the performance of the bicistronic vector in transient and/or stable reporter assays using $\mathrm{CHO}, 293$, NIH3T3, and CV-1 cells as hosts. We show the results for a double stable $\mathrm{CHO}$ cell line, for which induction ratios of more than three orders of magnitude are achieved.

\section{MATERIALS AND METHODS}

\section{Cell Culture and Transient Transfections}

CHO, NIH3T3, CV-1, and 293 cells were maintained in DMEM supplemented with $10 \%$ FBS with or without penstrep. Transient transfections were carried out using LIPOFECTAMINE $^{\mathrm{TM}}$ and optiMEM ${ }^{\mathrm{TM}}$ Reduced Serum Medium (both from Life Technologies, Rockville, MD, USA) accord-

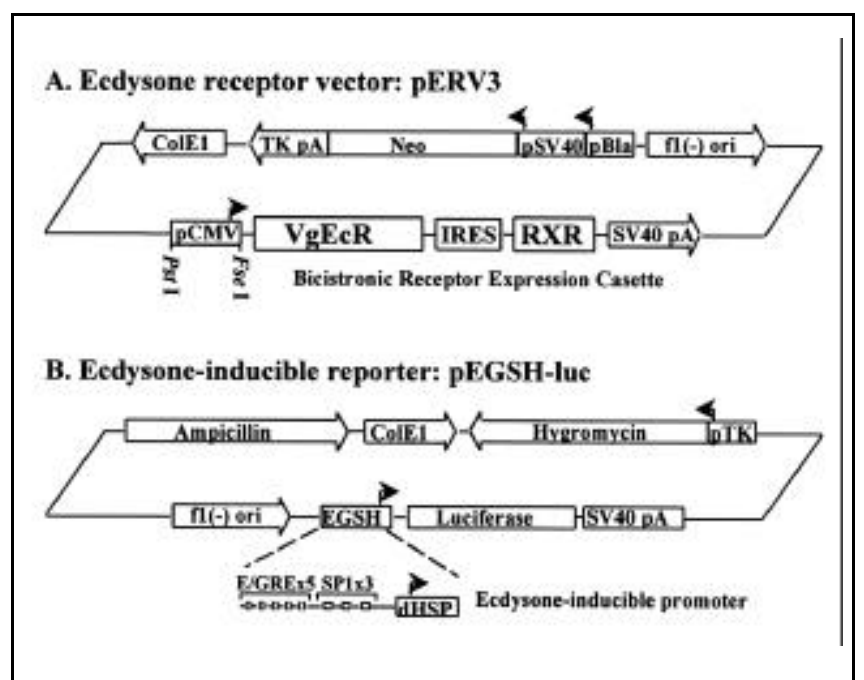

Figure 1. Vectors. (A) Map of pERV3. The vector is 8433 bp and has the GenBank ${ }^{\circledR}$ accession no. AF098284. The IRES is from the EMCV (1). The aminoglycoside phosphotransferase coding sequence (designated as Neo in the map) is expressed from the SV40 early promoter (pSV40) and the E. coli $\beta$-lactamase promoter for expression in mammalian cells and $E$. coli, respectively. pA, polyadenylation signal. (B) Map of the $\mathrm{pEGSH}-\mathrm{luc}$ reporter vector. The vector contains the coding sequence for the firefly luciferase gene that was inserted downstream of the ecdysone-inducible promoter (EGSH). The inducible promoter in this construct includes the $\mathrm{dHsp}$ flanked in the $5^{\prime}$ direction by three copies of the binding site for the transcription factor Sp1, followed by five copies of the binding site for the VgEcR-RXR heterodimer (E/GRE). ing to the manufacturer's recommendations. In all cases, the total amount of DNA transfected was brought up to $2 \mu \mathrm{g} / 35$ mm well with pBluescript ${ }^{\circledR}$ II DNA (Stratagene, La Jolla, CA, USA). Cells were incubated 5-24 h with the DNA-lipid complexes and then were allowed to recover for at least $4 \mathrm{~h}$, following the removal of the DNA-lipid complexes. Cells were then induced with muristerone A (murA), ponasterone A (PNA) (7), or an equivalent volume of ethanol for $20 \mathrm{~h}$, unless otherwise indicated.

\section{Vector Construction}

The vector $\mathrm{pERV} 3$ was constructed by first PCR-amplifying the $\mathrm{VgEcR}$ receptor from the vector pCMX-VgEcR (8), using primers that were engineered so that the resulting PCR product contained unique SpeI and FseI sites at the $5^{\prime}$ end, followed by the consensus Kozak sequence ACCATGG (5) in frame with the N-terminus of the VgEcR coding sequence (Figure 2). The $3^{\prime}$ end of the fragment contained the following unique restriction sites in $5^{\prime} \rightarrow 3^{\prime}$ orientation, downstream of the VgEcR termination codon: BstEII, PvuI, NotI, and MluI. The sites contained short spacer sequences so that contiguous sites could be efficiently digested. The VgEcR-encoding PCR fragment was then inserted into the NheI and $M l u \mathrm{I}$ sites of the plasmid pBKCMV to create the vector $\mathrm{pVgEcR/pBK-CMV}$. The 630-bp IRES sequence from the EMCV was PCR-amplified from the plasmid pCITE (Novagen, Madison, WI, USA) so that the resulting PCR fragment contained unique BstEII and $P v u I$ sites at the $5^{\prime}$ and $3^{\prime}$ termini, respectively. This fragment was inserted between the $B s t$ EII and $P v u I$ sites in $\mathrm{pVgEcR} / \mathrm{pBK}-\mathrm{CMV}$ to create the plasmid $\mathrm{pVgEcR/EMC/pBK-CMV.} \mathrm{The} \mathrm{RXR} \mathrm{recep-}$ tor coding sequence was PCR-amplified from the vector pCMX-hRXRa (8), with the resulting PCR product containing flanking $P v u \mathrm{I}$ and $N o t \mathrm{I}$ sites at the $5^{\prime}$ and $3^{\prime}$ ends, respectively. The RXR coding sequence was inserted between the $P v u \mathrm{I}$ and Not I sites of pVgEcR/EMC/pBK-CMV. The 450-nucleotide simian virus 40 (SV40) polyadenylation (polyA) sequence from the plasmid pCMVLacI was PCR-amplified, resulting in a product containing $N o t \mathrm{I}$ and $M l u \mathrm{I}$ sites at the $5^{\prime}$ and $3^{\prime}$ ends, and was inserted into the NotI and MluI sites of the RXR-containing vector, resulting in the vector $\mathrm{pERV}$. Finally, a unique PstI site was introduced at the $5^{\prime}$ border of the CMV promoter by site-directed mutagenesis using the QuikChange ${ }^{\mathrm{TM}}$ kit (Stratagene), resulting in the vector pERV3.

The vector $\mathrm{pEGSH}$ was constructed by first PCR-amplifying the large fragment between coordinates 468 and 3903 of the plasmid pCMVLacI, which contains the SV40 polyA site, the hygromycin resistance gene, the ColE1 origin, the $\beta$-lactamase gene, and the f1(-)ori. PCR was performed using Taq Plus $^{\mathrm{TM}}$ (Stratagene) with 10 cycles of amplification. One of the primers overlapped with and included the $B g$ III site at 468; the other primer included an NheI site engineered immediately upstream of the SV40 polyA site. A 535-nucleotide fragment from coordinates 1 to 535 from the vector pIND(SP1) (Invitrogen, Carlsbad, CA, USA), which contains five contiguous E/GRE binding sites, three SP1 sites, followed by a 248-nucleotide sequence containing the Drosophila minimal heat-shock promoter ( $\mathrm{dHsp})$ and $5^{\prime}$ untranslated region (UTR), was PCR-amplified in the presence of m5dCTP. The PCR product retained the $B g l$ II site at coordinate 1 and an Eaml104I site, a short spacer, and an NheI site in the $5^{\prime} \rightarrow 3^{\prime}$ orientation at its $3^{\prime}$ end. In addition, a C-to-G mutation was engineered at nucleotide 528 in this fragment. 
A. CHO

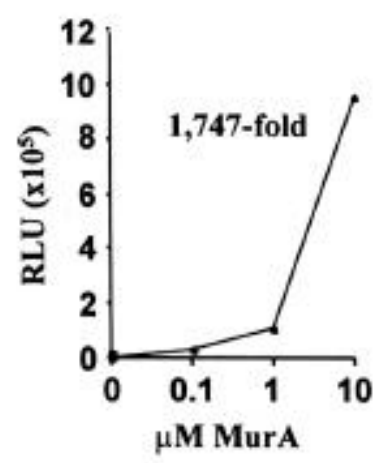

B. 293

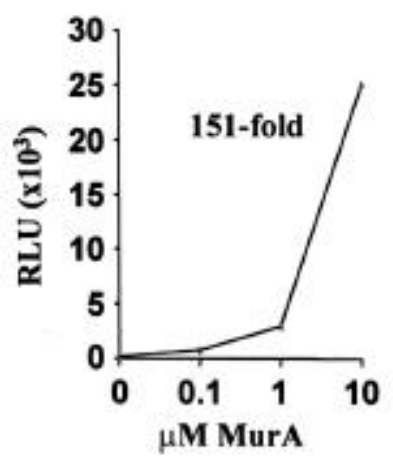

C. CV-1

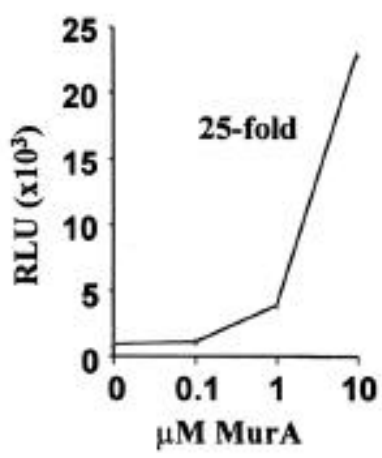

Figure 2. Performance of the pERV3 vector in transient assays. (A) $2 \times 10^{5} \mathrm{CHO}$, (B) 293 , or (C) CV-1 cells were co-transfected with 7 ng pEGSH-luc and $8 \mathrm{ng}$ pERV3 and induced for $20 \mathrm{~h}$ with the indicated amounts of murA. All results represent the average of duplicate samples.

The fragment was digested with $B g I I I$ and Eaml 104I and ligated to the BglII and NheI sites of the pCMVLacI "backbone" fragment to create the vector pTACO. Two Eaml 104I sites in pTACO, one corresponding to coordinate 7875 in pCMVLacI and one corresponding to coordinate 479 in the vector pIND(SP1), were destroyed using the QuikChange kit. Destruction of the latter Eam1 104I site also resulted in the insertion of a $\mathrm{C}$ residue between coordinates 457 and 458; both of the engineered changes and the $\mathrm{C}$ insertion fall within regions of the heat-shock promoter $5^{\prime}$ UTR that are not expected to affect the efficiency of the translation of the inserted protein coding sequence (6). In addition, a MunI site within the SV40 polyA, corresponding to position 4175 in the pCMVLacI sequence, was destroyed using the Chameleon ${ }^{\circledR}$ kit (Stratagene). The resulting plasmid was digested with Eaml 104I and NheI. Five overlapping oligonucleotides were hybridized together to create the multiple cloning site sequence from the upstream Eaml 104I site and the NheI site downstream of the T3 promoter (Figure 2B) and were inserted between the Eaml 104I and NheI sites of the vector to create the vector pEGSH. The vector pEGSH-luc was constructed by PCR-am plifying the firefly luciferase protein coding sequence; the resulting PCR fragment contained a Kozak consensus sequence at the $5^{\prime}$ end of the protein coding sequence and was flanked upstream by a unique KpnI site. The $3^{\prime}$ end contained a unique SalI site downstream of the luciferase termination codon. The fragment was cloned as a KpnI-SalI fragment into the mutiple cloning site of pEGSH.

\section{Production of Stable Cell Lines}

The production of cell lines stably transformed with the plasmids pERV3 or pEGSH-luc was performed using the Mammalian Transfection kit (Stratagene) in accordance with the recommended protocols. For each transfection, $20 \mu \mathrm{g}$ DNA were used. Following the removal of calcium phosphate precipitates, the cells were allowed to recover in complete medium for $24 \mathrm{~h}$ and were then split into a ratio of greater than or equal to 1:10. Twenty-four hours after the cells were split, $400-800 \mu \mathrm{g} / \mathrm{mL}$ G418 (pERV) or hygromycin (pEGSHluc) were added to the media. Drug-resistant colonies were

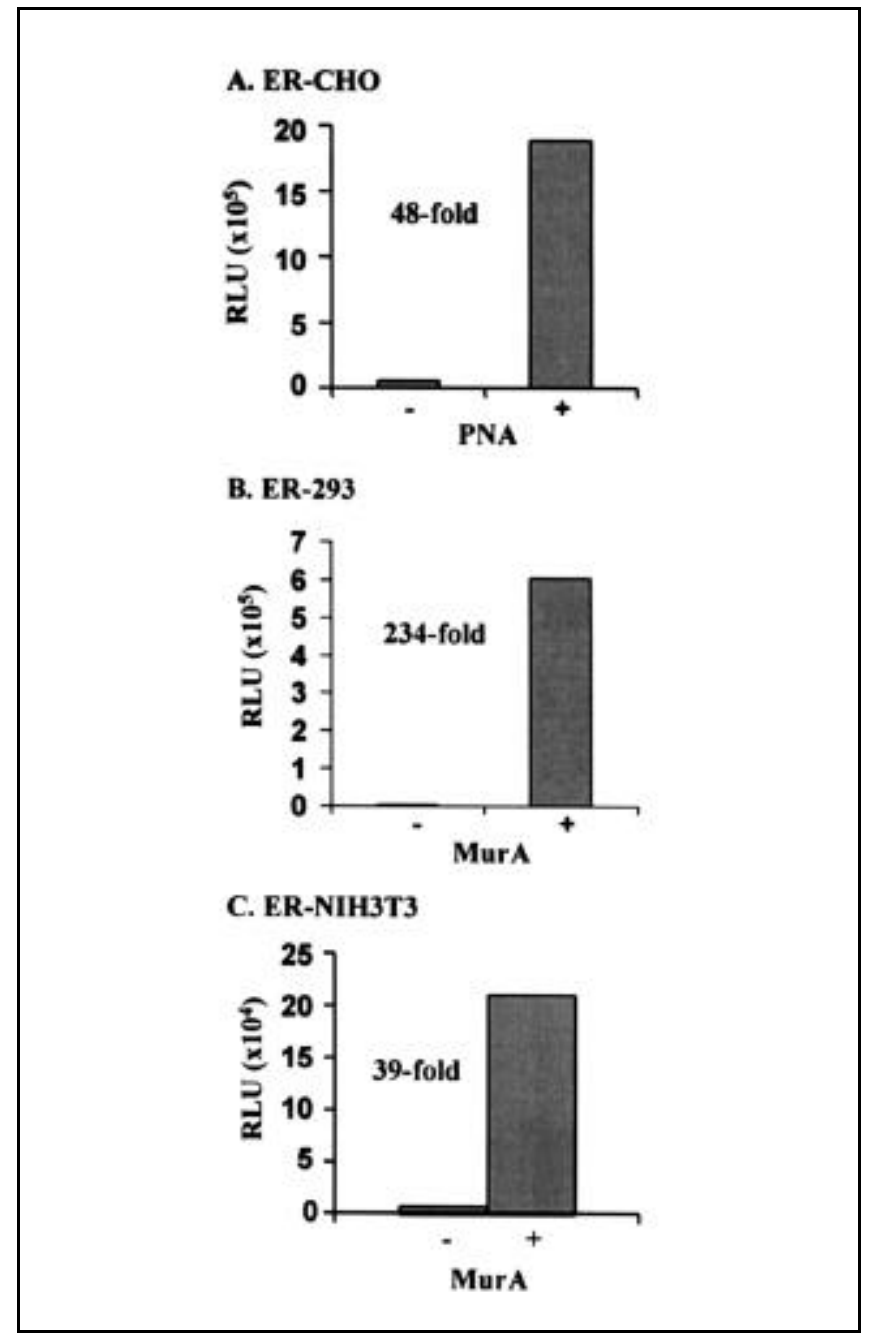

Figure 3. Evaluation of stable receptor-expressing cell lines. CHO, 293, or NIH3T3 cells were stably transformed with the vector pERV3, and expanded colonies were screened for cell lines that exhibited the highest inducer-dependent induction ratios following the transient transfection with $7 \mathrm{ng}$ pEGSH-Luc. The best of the pERV3-stable lines were designated (A) ERCHO, (B) ER-NIH3T3, or (C) ER-293, respectively. Cells were treated with either (A and C) 10 or (B) $5 \mu \mathrm{M}$ indicated inducers. 
isolated using cloning rings and expanded according to standard methods.

\section{Luciferase Assays}

All luciferase assays were carried out using the Luciferase Assay kit (Stratagene) according to the recommended protocol. Luciferase activity was measured for $10 \mathrm{~s}$, beginning $8 \mathrm{~s}$ after the extract and substrate were mixed, using a Tropix ${ }^{\mathrm{TM}}$ ILA-911 luminometer. Within each experiment, results were normalized to the protein concentration of the extract, as determined by the Coomassie ${ }^{\circledR}$-Plus Protein Determination assay (Pierce Chemical, Rockford, IL, USA).

\section{Western Blot}

Cells were washed twice in PBS before lysis. Cell pellets were resuspended in RIPA buffer $(50 \mathrm{mM}$ Tris- $\mathrm{HCl}$, $\mathrm{pH} 7.4$, $50 \mathrm{mM} \mathrm{NaCl}, 1 \%$ Triton $^{\circledR} \mathrm{X}-100,0.5 \%$ deoxycholate, $5 \mathrm{mM}$ EDTA, $1 \mathrm{mM}$ DTT, $0.2 \mathrm{mM}$ sodium vanadate, $25 \mathrm{mM} \mathrm{NaF}$, $10 \mathrm{mM}$ sodium pyrophosphate, $25 \mathrm{mM}$ glycerophosphate, 1 $\mathrm{mM}$ PMSF, $10 \mu \mathrm{g} / \mathrm{mL}$ aprotinin, and $10 \mu \mathrm{g} / \mathrm{mL}$ leupeptin). Protein determinations were carried out as previously described, and equivalent amounts of protein were fractionated by SDS-PAGE and electrophoretically transferred to nitrocellulose. Anti-luciferase polyclonal antibody (Biogenesis, San Diego, CA, USA) was detected using $\alpha$-rabbit IgG-horseradish peroxidase conjugate (Amersham Pharmacia Biotech, Piscataway, NJ, USA), followed by chemiluminescence using the ECL ${ }^{\circledR}$ kit (Amersham Pharmacia Biotech).

\section{RESULTS AND DISCUSSION}

\section{Evaluation of the pERV3 Vector in Transient Expression Assays}

To assess the performance of the pERV3 receptor expression vector, a set of transient expression assays were carried out using the reporter vector pEGSH-luc (Figure 2). This vector contains coding sequence for the firefly luciferase protein, which is inserted into a multiple cloning site downstream of an ecdysone-inducible promoter. $\mathrm{CHO}, 293$, or CV-1 cells were co-transfected with optimal amounts of pEGSH-luc and pERV3, and $24 \mathrm{~h}$ following transfection, the cells were induced with various amounts of the ecdysone analog murA for $20 \mathrm{~h}$ and then assayed for luciferase activity. As the results in Figure 2A indicate, an induction ratio of greater than three orders of magnitude was achieved in the $\mathrm{CHO}$ cells. Inducer-dependent inductions were also observed for the 293 and CV-1 cells, although maximum induction in these cell lines was approximately 40-fold lower than that observed for $\mathrm{CHO}$ cells, a result that is likely due, at least in part, to reduced transfection efficiency for these cell lines (data not shown; compare Figure $2 \mathrm{~B}$ and Figure $3 \mathrm{~B}$ ). The 6-fold lower induction ratio observed for CV-1 cells relative to 293 cells is due to increased uninduced expression of luciferase in this transient assay.

\section{Production of pERV3 Stable Cell Lines}

Next, we produced stable cell lines with the pERV3 vector using CHO, 293, or NIH3T3 cells as hosts. For each of the host cell lines, G418-resistant colonies were picked and expanded, transiently transfected with the pEGSH-luc reporter vector, and assayed for murA responsiveness. For the $\mathrm{CHO}$ cell isolates, eight out of nine isolates tested showed some induction above background, with two isolates showing inductions that were more than 10 -fold in this non-optimized primary screen. One of these clones, ER-CHO, exhibited a 53-fold murA induction and a 48-fold induction when retested with the closely related analog PNA (7) (Figure 3A). Sim ilarly, 10 of 11 293-cell isolates showed some level of murA induction. Two of the 10 clones, A9-293-11 and ER-293, showed inductions of 183- and 234-fold, respectively. For the NIH3T3 clones, 12 of 15 isolates showed an induction, with four clones showing inductions ranging from 19- to 26-fold. We achieved a 39-fold induction on retesting the clone ERNIH3T3 (Figure 3C), which exhibited a 26-fold induction in the primary screen.

\section{Production of Double Stable CHO Cell Lines}

In the absence of ligand, RXR heterodimers remain bound to their cognate promoters in complexes containing co-repressors and histone deacetylase, and the promoter is tightly repressed until ligand binding, at which time the promoter is transcriptionally activated $(2,9)$. Thus, in the natural chromosomal context, the promoter-bound heterodimer functions both as a transcriptional repressor in the absence of ligand and as a trans-activator during ligand binding. Therefore, we expected that stably integrated ecdysone-inducible plasmids would generally exhibit a lower uninduced background transcription and thus higher induction ratios, compared with the transient reporter assays previously described, for which a considerably larger number of free nuclear plasmids that are unbound by heterodimer might be expected to contribute to uninduced background expression.

To test the vectors in a double stable cell line, ER-CHO cells were stably transfected with pEGSH-luc, and hygromycin-resistant colonies were screened for both low uninduced background and high-level, inducer-dependent expression of luciferase activity. All of the 36 clones tested showed some level of induction above background, and eight of the clones exhibited induction ratios greater than 20 -fold. The results for one of the double stable clones, HSL-34, are shown in Figure 4. Initially, we wished to compare the ecdysone analogs murA and PNA for their ability to induce the cell line. As Figure 2A indicates, both inducers activate luciferase expression to comparable levels over a 100-fold range of inducer concentration, with a peak induction ratio of approximately 600 -fold at $10 \mu \mathrm{M}$ inducer. To more accurately assess the sensitivity of the system, we varied both the PNA concentration and induction time. The results in Figure $2 \mathrm{~B}$ indicate that dose-responsiveness was observed from $80 \mathrm{nM}$ PNA (4-fold; the lowest concentration tested) and was linear from 0.63 to $10 \mu \mathrm{M}$. In a time-course experiment, a 5-fold induction was achieved only $1 \mathrm{~h}$ after the addition of $10 \mu \mathrm{M}$ PNA, and a linear increase was observed up to $20 \mathrm{~h}$, at which time an induction ratio of 1030-fold was observed (Figure 2C). We also observed these induction kinetics in transient assays (data not shown). To more directly ascertain the control of protein expression in this cell line, we performed a western blot. Lysates from uninduced cells and cells induced with increasing amounts of PNA were fractionated by SDS-PAGE, blotted, 


\section{A. Inducer Comparison using HSL-34 Cell Line}

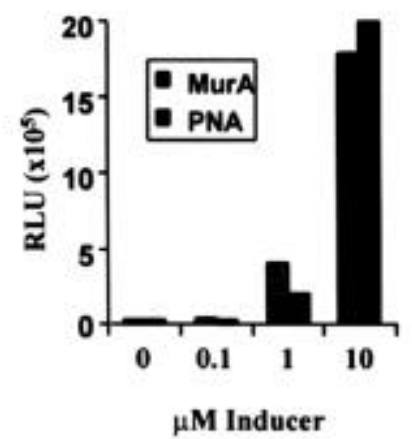

B. PNA Dose: Response in HSL-34 Cells

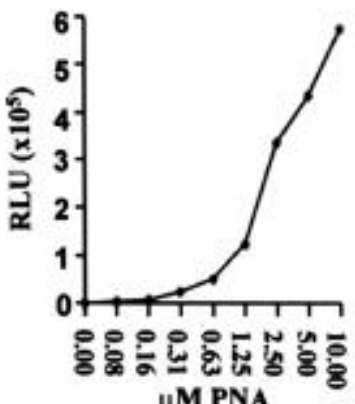

C. PNA Time-Course in HSL-34 Cells

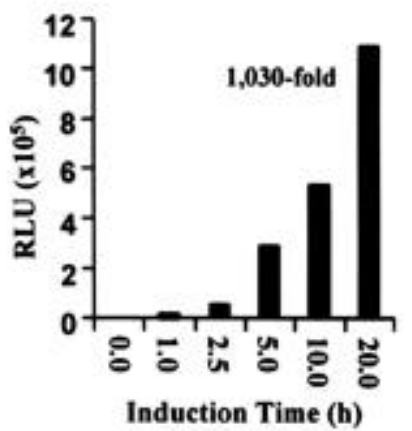

D. Western Blot

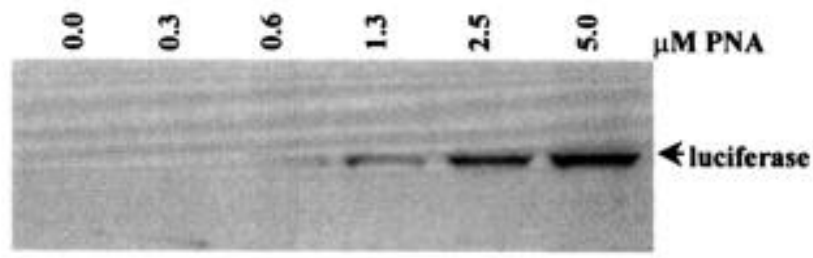

Figure 4. PNA dose-responsiveness and kinetics of induction for the double stable cell line HSL-34. All of the results in panels A-C represent the average relative light unit (RLU) value for duplicate samples. Inductions were carried out for $20 \mathrm{~h}$ unless otherwise indicated.

and probed with $\alpha$-luciferase polyclonal antisera (Figure 2D). No significant detectable luciferase is expressed in the uninduced extract, whereas a linear increase in signal is observed from $300 \mathrm{nM}$ to $5 \mu \mathrm{M}$ PNA. Taken together, these results indicate that precise control of gene dosage can be achieved in stable cells using pERV3 by varying either the PNA concentration or the time of induction. Furthermore, comparatively brief induction periods can result in low to moderate levels of gene expression.

We have recently constructed a version of the pERV3 vector that contains a multiple cloning site in place of the CMV promoter, an improvement that should expedite the production of a panel of pERV3-derived vectors containing a wide range of tissue-specific promoters for use in transgenic animals. In addition, co-expression of the receptors from a single transcript is essential for the adaptation of the ecdysone-inducible system to retroviral vectors, an improvement that will facilitate the delivery of the system to cells that are difficult to transfect by conventional means and should thus accelerate the production of transgenic animals.

\section{ACKNOWLEDGMENTS}

We thank Drs. Ronald M. Evans, Enrique Saez, (Salk Institute), and Joe Sorge for helpful discussions, and Grady Howe and Tim Sanchez for technical support.

\section{REFERENCES}

1.Borman, A.M., P. Le Mercier, M. Girard, and K.M. Kean. 1997. Com parison of picornaviral IRES-driven internal initiation of translation in cultured cells of different origins. Nucleic Acids Res. 25:925-932.
2.Chen, J.D., K. Umesono, and R.M. Evans. 1996. SMRT isoforms mediate repression and anti-repression of nuclear receptor heterodimers. Proc. Natl. Acad. Sci. USA 93:7567-7571.

3.Christopherson, K.S., M.R. Mark, V. Bajaj, and P.J. Godowski. 1992. Ecdysteroid-dependent regulation of genes in mammalian cells by a Drosophila ecdysone receptor and chimeric transactivators. Proc. Natl. Acad. Sci. USA 89:6314-6318.

4.Ghattas, I.R., J.R. Sanes, and J.E. Majors. 1991. The encephalomyocarditis virus internal ribosome entry site allows efficient coexpression of two genes from a recombinant provirus in cultured cells and in em bryos. Mol. Cell. Biol. 11:5848-5859.

5.Kozak, M. 1996. Interpreting cDNA sequences: some insights from studies on translation. Mamm. Genome 7:563-574.

6.McGarry, T.J. and S. Lindquist. 1985. The preferential translation of Drosophila hsp70 mRNA requires sequences in the untranslated leader. Cell 42:903-911.

7.McMorris, T.C. and B. Voeller. 1971. Ecdysones from gametophytic tissues of a fern. Phytochemistry 10:3253-3254.

8.No, D., T.-P. Yao, and R.M. Evans. 1996. Ecdysone-inducible gene expression in mammalian cells and transgenic mice. Proc. Natl. Acad. Sci. USA 93:3346-3351.

9.Saez, E., D. No, A. West, and R.M. Evans. 1997. Inducible gene expression in mammalian cells and transgenic mice. Curr. Op. Biotechnol. 8:608-616.

10.Sawicki, J.A., B. Monks, and R.J. Morris. 1998. Cell-specific ecdysone-inducible expression of FLP recombinase in mammalian cells. BioTechniques 25:868-875.

11.Schockett, P.E. and D.G. Schatz. 1996. Diverse strategies for tetracycline-regulated inducible gene expression. Proc. Natl. Acad. Sci. USA 93:5173-5176.

Address correspondence to Dr. Peter Vaillancourt, Applied Molecular Evolution, 3520 Dunhill Street, San Diego, CA 92121,USA.e-mail: pvaillancourt@amevolution.com

For reprints of this or any other article, contact Reprints@BioTechniques.com 